die Abmachungen nicht schriftlich festgesetzt worden. Um die Unzufriedenheit der Aerzte zu beseitigen, sollen nun Verhandlungeu mit der Berufsgenossenschaft gepflogen und deren Ergebnisse protokollarisch festgelegt werden. - Eine Vorlage des Mü: chener Bezirksvereins, daB Aerzte als Aufsichtsbeamte bel dir Gewerbeinspektiou angestellt werden sollen, wird in Oberbayern und Niederbayern angenommen, von Oberfranken, Mittelfranken und der Pfalz abgesetzt, weil die Motive der Antragsteller nicht übersandt worden sind, in den übrigen Kammern nicht verhandelt. - A uf das Recht, Sachrerständige zum Schied:gericht für Arbeiterversicherung vorzuschlagen, verzichtet die Kammer von Unterfranken schon seit einigen Jahren, die übrigen stellen eine Liste auf, doch will die oberbayerische Kammer in der nächsten Sitzung eine Anfrage an das Schiedsgericht beraten, wie sich dieses in Zukunft zu den Vorschlägen zu stellen gedenkt, nachdem die betreffende Aufstellung bisher seitens des Schiedsgerichts keine Beachtung gefunden hat. - Eine interessante Verhandlung knüpfte sich in der Kammer von Mittelfranken an eine Entscheidung des bayerischen Verwaltungsgerichtshofes, wonach die Annahme einer Krankheit und der Eintritt einer solchen "rein sachlich, d. h. ohne ausschlaggebende Rücksicht auf Meinung, Willen und Verhalten der Versicherten zu beurteilen ist". $\mathrm{Da}$ die so geforderte Feststellung des Begriffes der objektiven Hilfsbedürftigkeit Schwierigkeiten und Schwankungen unterliegt, sollen die Bezirksvereine aufgefordert werden, der Sache näher zu treten.

Die Brandenburg-Berlinische Aerztekammer hat sämtlichen

\title{
Standesangelegenheiten.
}

\section{Aus den Verhandlungen der bayerischen Aerztekammern im Jahre 1905.}

Die acht bayerischen Aerztekammern traten im Jahre 1905 am 23. Oktober zil ihren Sitzungen zusammen. Wie noch immer nicht anders zu erwarten, nahmen auch dieses Mal die Fragen, welche mit der sozialen Gesetzgebung zusammenhängen, einen breiten Raum in den Verhandlungen ein. Dabei ist es auffallend, daB die Begeisterung. mit welcher im Jahre vorher die bayerische wirtschafticlic Zentrale ins Leben gerufen wurde, vollständig verrauscht ist; die Zentrale hat schon zu existieren aufgehört, angeblich weil die ärztliche Organisation weit genug vorgeschritten ist, um ihrer entbehren zu können. Leider haben die rom Aerztevereinsverbande auf die Münchener Anregung hin ausgegebenen Verpfichtungsscheine auch in Bayern im allgemeinen nicht mehr Unterschriften gefunden als in Norddeutschland. - Der Antrag, es möge die Erteilung eines Lehranftrages für Unfallknnde, Sozialgesetz. gebung aud Gntachterwesen an den Landesuniversitäten, speziell zunächst in München, befürwortet werden, wurde in drei Kammern, da er zu spät eingelaufen, nicht verhandelt; die Kammer der Oberpfalz und von Regensburg nahm ihn mit großer Sympathie entgegen, stimmte aber aus dem Grunde nicht zu, weil sie sich nur von einem gemeinsamen Vorgehen aller bayerischen Kammern einen Erfolg versprach; die übrigen vier Kammern nahmen ihn einstimmig an. - Zufällig ist durch den veröffentlichten Militäretat bekannt geworden, daß regierungsseitig beabsichtigt ist, eine Militïrkrankenkasse nach Art der Post- und Bahnkrankenkasse ins Leben zu rufen, die mindestens 2000 Mitglieder zählen wird. Obwobl die Verhältnisse der Bahn- und Postkasse wegen der durch sie hervorgerufenen Spaltung der Münchener Aerzteschaft mehr als nützlich öffentlich besprochen worden sind, hielt man es nicht für nötig, vor der Gründung der neuen Kasse die Aerzte zu befragen, die sich natürlich trotzdem mit der Angelegenhoit beschäftigten. In drei Kammern kam die Frage nicht zur Verhandlung, da sie nicht zeitig genug bekannt geworden war, die übrigen fünf beschlossen, mit aller Energie für die Einführung der freien Aerztewahl bei der Militärkrankenkasse einzutreten. - A uf Veranlassung des Staatsministeriums haben die Kammern sich über ein Gesuch der dem Landesversicherungsamt unterstehenden (acht) Berufsgenossenschaften auszusprechen, wonach die Sätze der Gebührenordunng für amtsärztliche Dienstleistungen bei Ausstellnng von Unfallattesten herabgesetzt werden sollen. Die gewünschte Ermüigung wird nicht nur in sämtlichen Kammern abgelehnt, sondern auch in der großen Mehrzahl derselben ein Antrag des Vereins der bayerischen Psychiater angenommen, daß in der besagten Gebührenordnung der Zusatz Aufnahme finde: „für besonders zeitraubende Gutachten 30-75 Mark". Im AnschluB an diesen Punkt kommt in der Kammer von Mittelfranken zur Sprache, daB die dortige land- und forstwirtschaftliche Berufsgenossenschaft den von einer Kommission der Kammer mit ihr abgeschlossenen Vertrag bezüglich der Honorierung von Gutachten in nicht zu billigender Weise, natürlich zum Nachteil der Aerzte, ausgelegt habe. Leider waren bayerischen Kammern den Bericht ihrer Kommission für die Revision der Bestimmungen des Strafgesetzbnches über die Ausübung der Heilkunde zugesandt. Die Angelegenheit wurde außer in Oberbayern, wo die Verhandlung darüber in der nächsten Sitzung stattfinden soll, und in Unterfranken überall beifallig besprochen und dazu der Beschluß gefaß3t, die Schrift der Regierung zu überreichen, mit der Bitte um Mitteilung, wie weit die Staatsregierung gewillt ist, diese berechtigten und notwendigen $\mathrm{Be}$ strebungen der Aerzte geeigneten Ortes zu unterstützen.

Die ärztlichen Fortbildungskurse haben auch in Bayern vielen Beifall gefunden, aber da die Kosten für den Aufenthalt in der Universitäts- oder Fortbildungsstadt und für die Besoldung eines Vertreters manchen Arzt gegen seinen Willen von der Teilnahme abhalten dürfte, so soll an den Landtag um die Gewährung von Mitteln für die sich an den Kursen Beteiligenden petitioniert werden. Hiermit erklärten sich die Kammern einverstanden. Nur Schwaben. Neuburg und Oberfranken lehnten ab, das letztere mit der Begründung, daß ein Bedürfnis materieller Unterstützung nicht vorhanden sei und die Erstrebung einer solchen vom Standesinteresse aus bedenklich erscheine. $\mathrm{DaB}$ aber die Einkommensverhältnisse auch in Bayern keine glanzenden sind, geht daraus hervor, dafs die Bezirksvereine von allen Kammern dringend darauf hingewiesen werden, für die Zuwendung von zahlreichen Beiträgen an die Witwenkasse nach besten Kräften zu sorgen.

Mit Einmütigkeit wurde in allen Kammern die Verschleppung getadelt, welche seitens der Staatsregierung bezüglich der Verleihnug der Korporationsrechte an die Bexirksvereine beliebt worden ist. (Die Beschwerde hat inzwischen Erfolg gehabt; siehe diese Wochenschrift S. 72 . D. Red.)

In mehreren Kammern wurde über Desinfektionsfragen verhandelt. In Oberbayern wurde ein Antrag, daß die Hebammen Gummihandschuhe gebrauchen sollen, vor der Abstimmung zurückgezogen; dagegen soll die Regierung ersucht werden, die Gebühren der Hebammen für die Desinfektion bei einer Geburt von 1 Mark auf $1 \frac{1}{3}$ Mark zu erhöhen; die Einführung einer Desinfektionsordnung wird in der nächsten Kammertagung besprochen werden. In Oberfranken wird eine bereits im vorigen Jahre erfolgte Eingabe wegen staatlicher Regelung des Desinfektionswesens nochmals beschlossen. In der $\mathrm{Pfalz}$ hat dieses grobe Fortschritte aufzuweisen, indem in allen Distrikten Dampfdesinfektions- und For malinapparate vorhanden sind, die von geschulten Desinfektoren bedient werden. In Ludwigshafen steht man davor, die Vornahme der Desinfektionen unter die direkte Leitung eines Arztes zu stellen. - Um die Einschleppung von Infektionskrankheiten zu verhüten, richtet die Kammer von Unterfranken an die Regierung das Ersuchen, dahin zu wirken, daß die benachbarten ausländischen Gemeinden bei Ausbruch von Epidemien, wie Typhus etc., die nächsten bayerischen Behörden schleunigst benachrichtigen.

Da die ärztlichen Standesinteressen und die sanitären Fragen überall die gleichen sind, kam in den einzelnen Kammern natürlich auch eine Menge von Gegenstanden zur Debatte, die uns von Norddeutschland her sattsam bekannt sind. So wurde in der Kammer von Oberbayern über die Bestrebungen der Zahntechniker auf höhere Stellung verhandelt, dieser Gegenstand aber bis zar 
nächsten Sitzung vertagt. Der von Leipzig ausgehende Antrag, es solle neben der Reichsarzneitaxe auch eine Reichshandverkanfstaxe eingefubrt werden, wurde in Schwaben angenommen, dagegen in Niederbayern abgelehnt. - Fegen die Zulassung von Kandidaten die das Probejahr noch nicht beendet haben, zar Vertretung ron Aerztelı sprach sich auch die pfälzische Kammer aus. - In Oberfranken wird die Regierung ersucht, eine Enquete ïber die Zolleinnahmen ans importierten Geheimmitteln rorzunehmen, welche als Kampfmittel gegen die Kurpfuscher zur Verwendung kommen soll. - Die Kammer von Unterfranken hat den Wunsch, daß die Regierung vor Verleihung offizieller Stellen über die Personalien der Kandidaten bei der betreffenden Aerztekammer Erkundigungen einziehe. In derselben Kammer wird eine prinzipiell wichtige Entscheidnng mitgeteilt, wonach ein beamteter Arzt in seiner Tätigkeit als praktischer Arzt und als Mitglied eines ärztlichen Bezirksvereins den Satzungen sich ebenso zu unterwerfen hat wie jeder andere Arzt.

Es ist begreiflıch, da 3 in der oberbayerischen Kammer die Unstimmigkeiten zwischen dem Bezirksverein und dem neuen Standesverein in München wiederholt gestreift wurden Mit Genugtuung kann man feststellen, da $B$ die Gegegensätze nicht mehr so scharf herrortreten, wie es ror wenigen Monaten der Fall war, und so darf man hoffen. daß Terhandlungen, welche der Vorsitzende auf Beschluß der Kammern domnächst mit den beiden Münchener Parteien in die Wege leiten soll, von Erfolg gekrönt sein werden. L. Henius (Berlin). 